Théologiques

Théologiques

\title{
La Jérusalem souterraine du temps d’Ézéchias
}

Le tunnel de Siloé

\section{Maria Gorea}

Volume 21, numéro 1, 2013

L’archéologie et la Bible

URI : https://id.erudit.org/iderudit/1025470ar

DOI : https://doi.org/10.7202/1025470ar

Aller au sommaire du numéro

\section{Éditeur(s)}

Faculté de théologie et de sciences des religions, Université de Montréal

\section{ISSN}

1188-7109 (imprimé)

1492-1413 (numérique)

Découvrir la revue

\section{Citer cet article}

Gorea, M. (2013). La Jérusalem souterraine du temps d’Ézéchias : le tunnel de Siloé. Théologiques, 21(1), 97-116. https://doi.org/10.7202/1025470ar

\section{Résumé de l'article}

L'histoire de la découverte à Jérusalem d'un système hydraulique complexe sous la colline méridionale où fut creusé le tunnel de Siloé et de l'une des inscriptions hébraïques de la plus haute importance pour cette région avare en documents de nature épigraphique illustre de quelle manière une série de faits, dont certains fortuits, s'est posée à l'origine d'une reconstitution qui a mobilisé et continue de le faire des spécialistes du terrain et ceux du texte biblique, en brisant les barrières disciplinaires. 


\title{
La Jérusalem souterraine du temps d'Ézéchias
}

\section{Le tunnel de Siloé*}

\author{
Maria Gorea** \\ Langues sémitiques anciennes \\ Université Paris VIII (France)
}

\section{L'archéologie à la rencontre des sources textuelles}

La philologie, conçue comme un instrument antidogmatique, nécessaire au libre examen des textes anciens, s'est accompagnée, à l'époque moderne, d'une investigation sur les lieux d'élaboration des textes, se transportant dans une plus étroite proximité des faits et des vestiges. C'est une démarche qui, sous les apparences d'une vénération du passé biblique, se voulait critique, ouverte à l'enquête historienne et même au doute et dont le but était celui de confronter les données textuelles au relevé topographique et au matériau épigraphique. Une vue d'ensemble permettait d'organiser, à partir de parcelles, certes, lacunaires, une synthèse intelligible d'une histoire fondée sur documentation accessible la plus large et variée, quoique provisoire, susceptible d'être complétée ou révisée à mesure de l'accroissement des trouvailles. L'acte d'interprétation des données recueillies n'est ainsi plus subordonné de façon inconditionnelle à l'autorité des Écritures, mais celles-ci contribuent à la reconstitution de l'histoire conjointement aux vestiges tangibles, en même temps que leur propre aspect matériel

\footnotetext{
* Je tiens à remercier le relecteur anonyme de cet article pour ses remarques précieuses, qui m’ont permis, je l'espère, d'apporter des améliorations à ces quelques pages.

* Maria Goréa est Maître de conférences à l'Université Paris VIII (France) où elle enseigne l'hébreu et l'araméen anciens, la littérature biblique et les antiquités. Ses recherches actuelles portent notamment sur l'épigraphie et la philologie araméennes. Elle a récemment publié (2010) «Considérations sur la politisation de la religion à Palmyre et sur la dévotion militaire des Palmyréniens de la province romaine de Dacie », Semitica et Classica, 3, p. 125-162
} 
reçoit des disciplines et des méthodes objectives une configuration plus précise et un relief plus net.

Continuant la tradition de l'orientalisme savant des Lumières, la communauté scientifique française et européenne lui ont donné, tout le long du $\mathrm{XIX}^{\mathrm{e}}$ siècle, une profondeur historique, en s'impliquant à élaborer un savoir colonial fondé sur les sciences sociales et en multipliant les recherches topographiques et géographiques au Levant. En Syrie-Palestine, les prospections de terrain furent entreprises d'abord dans des buts militaires et stratégiques par les officiers de la force européenne et de la Couronne britannique. L'intérêt diplomatique et militaire des européens, préoccupés, dès les années 1830, à arrêter l'expansion égyptienne au Proche-Orient et à réinstaller le régime ottoman en Syrie-Palestine, afin de permettre le contrôle de la Méditerranée et des routes menant à l'Inde, s'accompagnait de considérations géo-religieuses et d'une préoccupation pour la question des origines. Par ailleurs, le sauvetage du patrimoine matériel grâce à l'archéologie, prôné par les scientifiques, était difficile à distinguer d'une campagne de chasse aux antiquités destinées à enrichir les collections européennes des musées fondés quelques décennies auparavant ${ }^{1}$.

La découverte de la Jérusalem biblique, dont — disons-le de suite — peu de vestiges subsistent, est le résultat d'une longue recherche, dont les buts s'étaient vus préciser dès la seconde moitié du XIX ${ }^{\mathrm{e}}$ siècle dans un climat d'émulation parmi les européens présents au Proche-Orient. On s'y intéressait déjà à l'époque romantique, lorsque le goût marqué pour les ruines

1. Le British Museum fut ouvert au public dans les années 1750, le Musée Napoléon, à Paris, en 1803, et la Glyptothèque de Munich en 1830. L'intérêt purement scientifique de ceux qui voyageaient en Grèce et en Orient, avec l'intention d'étudier les antiquités sur place, s'accompagnait d'un désir de les collectionner. Les premiers à constituer des collections de vases, statues et membra disiecta des temples antiques grecs et romains, mais aussi d'antiquités égyptiennes et celtiques, furent des diplomates européens auprès de la Sublime Porte, dont les plus connus sont le comte de Choiseul et lord Elgin. Marie Gabriel Florent Auguste, comte de Choiseul-Gouffier, nommé ambassadeur du Roi à Constantinople en 1784, écrivait à Louis François Sébastien Fauvel, peintre, antiquaire, archéologue à ses frais, puis, après 1802, viceconsul de la France à Athènes: "Enlevez tout ce que vous pourrez, ne négligez aucune occasion de piller dans Athènes ou dans son territoire tout ce qu'il y a de pillable. [...] N'épargnez ni les morts ni les vivants. » (BNF, ms. fr. 22873, fol. 164, lettre de Choiseul à Fauvel datant du 14 février 1789, citée par Zambon 2006, 4). Ce commerce lucratif, mené avec la complicité des autorités turques, procurait des revenus confortables aux détenteurs des objets et des honneurs, parfois académiques, à ceux qui, à travers un réseau de connaissances, facilitaient leur acquisition par les musées, vers lesquels affluaient des caisses remplies d'antiquités. 
et l'intérêt esthétique, l'attrait pour les monuments détruits et pour l'anéantissement qu'opère le temps ou les hommes étaient liés aux bouleversements politiques. Si les récits de voyageurs du XIX ${ }^{\mathrm{e}}$ siècle, qui présentent Jérusalem comme une cité en ruines et décrivent la vétusté et l'insalubrité de ses bâtiments, ont contribué à susciter et à entretenir le goût pour les orientalia, ces publications offraient aux lecteurs une image encore romantique des lieux bibliques, même si on leur reconnaît aujourd'hui une qualité de témoignage d'un état antérieur à l'urbanisation que ces lieux ont connues depuis ${ }^{2}$.

Lorsque le Palestine Exploration Fund fut fondé en 1865, marquant le début des fouilles et des recherches systématiques dans le domaine de l'archéologie et de la topographie à Jérusalem et en «terre sainte », d'autres sociétés savantes ou institutions, la Dilettanti Society, à Londres (fondée en 1734), l'Institut de correspondance archéologique de Rome (fondé en 1829), l'École française d'Athènes (créée en 1846), menaient des prospections de terrain depuis déjà quelques décennies, en Italie, en Dalmatie, en Grèce, dans les îles égéennes et en Ionie, mettant en œuvre la méthode archéologique prônée déjà par Jacob Spon, consistant à joindre à l'étude des sources textuelles les observations sur le terrain et l'exploitation systématique des inscriptions ${ }^{3}$. En même temps, les grandes civilisations du Proche-Orient étaient progressivement révélées aux archéologues ${ }^{4}$. Pour ce

2. En témoignent l'ouvrage illustré de lithographies de David Roberts, Carnet de voyage: Égypte, Syrie et Terre sainte, les descriptions des ruines et des lieux de Jérusalem et de la «terre sainte", publiées en français par Victor Guérin, dont l'ouvrage (in folio) est paru chez Plon en deux volumes en 1882 et 1884 (La Terre sainte. Ses souvenirs, ses sites, ses monuments; La Terre sainte [deuxième partie]. Liban, Phénicie, Palestine occidentale et méridionale, Pétra, Sinaï, Égypte).

3. Dans la préface de ses Miscellanea eruditae antiquitatis sive supplementi gruteriani. Liber primus, Spon $(1679,1-2)$ décrit ce que devrait être la discipline archéologique (Archaeographia), secondée par la numismatographia, l'epigrammatographia, la glyptographia et la taphographia.

4. Les fouilles de A. H. Layard (1845-1847; 1849-1851) à Nimrud/Kalhu (Kălah, dans Gen 10,11.12), l'exhumation (en 1846) de l'obélisque de Salmanasar III (858-824), aujourd'hui au British Museum, racontant ses campagnes, où est également fait mention de "Jéhu $(I a-u-a)$, fils de Omri (mār Hu-um-ri-i)», ainsi que la découverte des bibliothèques d'Assurbanipal [668-627] à Ninive (palais nord, fouilles débutées en 1842 à l'initiative de P.-É. Botta, alors agent consulaire de France à Mossul), puis à Khorsabad, pour n'en citer que deux d'une longue série de découvertes. Les monuments d'Égypte faisaient également l'objet d'études et de fouilles systématiques dont les travaux étaient soutenus par des fondations qui organisaient les chantiers, récoltaient et fournissaient les fonds nécessaires pour les entreprises archéologiques, se chargeaient de publier les résultats et, souvent, organisaient le transfert d'objets 
qui était de la Palestine, il ne fallait pas s'attendre à des découvertes épigraphiques ou iconographiques aussi spectaculaires qu'en Égypte ou en Mésopotamie. Aniconisme d'une part, modestie des productions écrites, de l'autre. Aussi les chercheurs se sont-ils plongés dans les investigations archéologiques, topographiques, géologiques et de la géographie physique, ainsi que dans l'étude des coutumes de cette région. Des officiers du Corps of British Royal Engineers, des cartographes et des savants déployés sur le terrain arpentaient Jérusalem, prenaient des mesures et corrigeaient les cartes. Les premières cartes détaillées de la région ont été élaborées avant que l'intérêt des archéologues ne se focalise sur Jérusalem même, où une première campagne scientifique (1864) procédant à la triangulation et au nivellement topographique a abouti à la publication, en 1865, par l'Ordnance Survey of Jerusalem (Wilson 1865), de plans détaillés (1. Notes) établis par les officiers du génie royal anglais en vue d'élaborer un projet d'adduction d'eau.

Le Palestine Exploration Fund avait décidé d'envoyer à Jérusalem deux savants, le capitaine Charles Wilson et son lieutenant, Charles Warren, afin de situer des lieux bibliques. Pour le faire, ils se sont servis des données de la Bible qu'ils ont complétées par les descriptions qu'en donne Flavius Josèphe. Warren fut notamment chargé d'explorer les côtés du Haram, mais le firman du pasha Nassif excluait l'esplanade du Temple de ses chantiers possibles. Il a ainsi procédé au dégagement du tunnel de Siloé, dernier d'une série de tunnels et installations liés à la source du Ghihon 5 .

Après la clôture de la première campagne du Survey à Jérusalem et tandis que l'on s'intéressait à d'autres régions palestiniennes, à Jérusalem, dans les années 1870-1880, l'architecte et missionnaire allemand Conrad Schick explorait, pour le compte de la Société allemande pour l'étude de la Palestine ${ }^{6}$, les vestiges de la vieille ville, notamment les installations hydrauliques souterraines du $\operatorname{Haram}^{7}$. Il fut aussi à l'origine d'une des plus impor-

trouvés en fouilles vers les musées européens, pillages qui avaient déjà une tradition, si l'on songe à tout ce que l'armée napoléonienne, le consulat britannique et divers aventuriers avaient déjà exporté d'Égypte notamment.

5. Wilson a reproduit un plan de Jérusalem en 1865 et, en 1867 , le lieutenant Warren a commencé ses fouilles, publiées dans un volume intitulé Underground Jerusalem (paru en 1876).

6. Deutscher Verein zur Erforschung Palästina's, fondée en 1877, à Leipzig.

7. Installé à Jérusalem depuis 1847 , Schick commença à publier ses observations techniques et dessins dans la revue du Deutscher Palästina-Verein (Schick 1878), mais aussi dans le Palestine Exploration Fund Quarterly Statement (ses premiers rapports datent de 1877), quelques années avant la découverte de l'inscription. Une brève 
tantes découvertes épigraphiques à Jérusalem, dans le tunnel creusé sous la colline sud-est de l'Ophel, reliant la source du Gihon ${ }^{8}$ à la piscine de Siloé (Birket Silwān): une inscription en anciens caractères hébraïques. Bien avant la découverte en 1880 de l'inscription hébraïque du tunnel de Siloé, celui-ci avait déjà fait l'objet de recherches de la part d'archéologues et ingénieurs ${ }^{9}$, mais après la publication, en 1880, de l'inscription, le tunnel de Siloé a fait l'objet d'investigations plus systématiques ${ }^{10}$.

\section{La découverte de l'inscription hébraïque du tunnel de Siloé}

En juin de l'année 1880, au hasard d'une heureuse glissade dans les eaux du tunnel, un jeune garçon, élève de Schick, Jacob Eliahou Spafford ${ }^{11}$, découvrait des lettres gravées superficiellement, alors qu'une grande partie de la surface inscrite de la roche se trouvait encore sous l'eau (Sayce 1881b, 141142). Schick en mesura toute l'importance et ne tarda pas à en faire l'annonce auprès du Deutscher Palästina-Verein et du Palestine Exploration Fund dans laquelle il publia un rapport ${ }^{12}$. Lorsque l'on trouva l'inscription,

campagne de fouilles allemande a été dirigée, en 1881, entre le Haram et la piscine de Siloé par Hermann Guthe.

8. Connue aussi sous les noms de «source de la Vierge » et 'Ayin Umm al-Darağ.

9. Il avait été inspecté, dès 1838, par Edward Robinson (1794-1863); par le savant suisse Titus Tobler (1806-1877), se trouvant à Jérusalem en 1845, 1846, 1865, qui en explora les sources et améliora les premiers plans de Jérusalem (qui reprennent ceux des lieutenants Aldrich et Symons, de 1841) (Robinson 1841; Tobler 1853, 73-87). En 1866, Warren avait fait les premières mesures de la longueur du tunnel, suivi de l'archéologue anglais W. F. Birch, qui, dans les années 1870, explorait les structures de Jérusalem pour le compte du même PEF (Birch 1880).

10. Bliss et Dickie identifièrent les restes d'une église byzantine dans la piscine de Siloé remontant à l'impératrice byzantine Eudoxie († c. 460), sur le lieu de commémoration de la guérison de l'aveugle de l'Évangile (Jn 9,7; Bliss et Dickie 1898, 132-210). Après le dégagement du tunnel lors de l'expédition Montagu B. Parker, en 1911, Vincent l'a étudié et mesuré à nouveau, obtenant une longueur de 533,10 m (Vincent 1911). Dans les années 1923-1924, Weil a fouillé le Canal IV, en partant de la piscine de Siloé vers la Vallée du Cédron (Weil 1947, 57-96).

11. Orphelin, né de parents juifs sépharades à Ramallah, le jeune Jacob David Eliahou (1864-1932), a été pris en charge par la London Jews Society de Jérusalem, avant de devenir, deux ans après la découverte de l'inscription et grâce à cet événement, le fils adoptif d'Anna et de Horatio Gates Spafford, les fondateurs de la Colonie américaine de Jérusalem (Fonds de manuscrits et photographies de l'American Memory Home, John D. Whiting Papers, Prints \& Photographs Division, Library of Congress).

12. Livraison d'octobre du Palestine Exploration Fund Quarterly Statement de 1880, aux p. 193-194, et Schick (1880). 
l'écriture phénicienne était déjà bien connue dans les milieux savants ${ }^{13}$. La découverte était de taille et l'on commença à s'empresser autour de cette inscription, pour laquelle la stèle moabite de Mésha, révélée au monde savant une dizaine d'années plus tôt, servait de terme de comparaison. Ce ne fut qu'en janvier de l'année suivante que Schick put en faire un facsimilé et photographier l'inscription à la lumière des lampes au ruban de magnésium, après que des travaux eurent été menés pour baisser le niveau de l'eau et la construction d'une plateforme en bois ${ }^{14}$. Au mois de février de l'année 1881, une autre copie de l'inscription fut établie, à la lumière de

13. C'est à Jacob Rhenferd (Periculum phoenicium: sive litteraturce phoenicice, quce late olim per Asiam, Africam et Europam patuit, eruendae specimen, Franequerae, 1706, in $4^{\circ}$, réimp. 1713, 1722), à Bernard de Montfaucon (Paleographia graeca, Paris, 1708, notamment p. 118-133), à l'abbé Barthélemy ( «éflexions sur quelques monuments phéniciens et sur les alphabets qui en résultent ", Mémoires de littérature tirés des registres de l'Académie royale des Inscriptions et Belles-Lettres XXX, 1764, p. 405-427) et à François Perez Bayer, archidiacre de Valence (De numis hebraeosamaritanis, Valence, 1781 , in $4^{\circ}$; Numorum hebraeo-samaritanorum vindiciae, Valence, 1790 , in $4^{\circ}$ ) que l'on doit les premières connaissances sur l'alphabet phénicien et punique (pour ce dernier, en 1824, Lindberg publiait son Commentatio de numis Punicis Sextorum olim Canacae et Concanae tributis, Hauniae, J. H. Schulz, et, en 1828, Hendrik Arent Hamaker ses Miscellanea phoenicia sive comentarii de rebus phoenicum, quibus inscriptiones multae lapidum ac nummorum, nominaque propria hominum et locorum, explicantur, item punicae gentis lingua et religiones passim illustrantur, Leiden [Lugduni Batavorum], Luchtmans, in $4^{\circ}$ ). Depuis le mémoire de l'abbé Barthélemy où il avait étudié l'inscription bilingue, phénico-grecque, du cippe de Malte (CIS [Corpus inscriptionum semiticarum] I, 122) et depuis ses travaux rassemblés en 1821 (Euvres complètes de J.-J. Barthélemy, Paris, t. 4), la découverte, en janvier 1855, à Saïda, du sarcophage d'Eshmunazor II (par Durighello) et celle, plus récente, en 1868, de la stèle de Mésha (avec les premières publications en 1870) avaient permis d'élucider certains points restés obscurs dans l'étude de l'écriture phénicienne (Meier, Die Grabschrift des sidonischen Königs Eschmun-ezer übersezt und erklärt [Abhandlungen der Deutschen Morgenländischen Gesellschaft, 4/4], Leipzig, 1866). Sur les recherches concernant les inscriptions phéniciennes jusqu'à l'époque du mémoire de l'abbé Barthélemy, voir Gesenius (1837), Scripturae linguaeque phoeniciae monumenta, Leipzig, Vogel, p. 1-2.

14. Afin de dégager l'inscription, alors immergée dans l'eau, et permettre ainsi que des relevés en soient pris, Schick avait suggéré de baisser le niveau de l'eau dans le tunnel. T. Chaplin fut chargé de récolter les fonds nécessaires à ces travaux et, dès le mois d'août de l'année 1880, le Comité de la Palestine Exploration Fund à Londres avait reçu de Jérusalem une première copie imparfaite de l'inscription. D'autres copies ont commencé à circuler, fruit d'une collaboration étroite entre les équipes allemande et anglaise (Sayce 1881a, 69). Un dessin d'après une copie faite par Schick fut publié en 1881 par Kautzsch dans Zeitschrift der Deutschen Morgenländischen Gesellschaft 4/1-2, pl. 4. 
chandelles, par Archibald H. Sayce, secondé par John Slater. La lecture n’y était que partielle ${ }^{15}$. La première transcription de Sayce sera vivement critiquée par Emil Kautzsch ${ }^{16}$, alors professeur à Tübingen, en même temps qu'il livrait une nouvelle lecture de l'inscription, créant un début de polémique et un échange d' "amabilités » entre les deux institutions, l'allemande et l'anglaise (Kautzsch 1881, 264 et 266). L'une comme l'autre revendiquait le droit de publier et d'exploiter des informations recueillies ${ }^{17}$. Des polémiques autour de la paternité des meilleures restitutions ou lectures apparurent également.

Côté allemand, une autre copie avait été réalisée au mois de mars 1881 par le savant Hermann Guthe, alors en Palestine, qui en avait fait plusieurs

15. Le fac-similé avait été mis à la disposition du comité de la Palestine Exploration Fund, à partir duquel, en avril, Sayce a tenté une première lecture (Sayce 1881a, 70). La description qu'en donne Sayce précise que: "Below the inscription comes an ornamental finish in the shape of two triangles, which rest upon their apices, with an angle between them similarly resting upon its apex» (p. 71). Le dessin de ces signes sera donné par Sulley, dans le Palestine Exploration Fund Quarterly de la livraison d'octobre de la même année, p. 297. Un second facsimilé et une lecture améliorée seront publiés par Sayce lui-même dans la livraison de juillet du même numéro (Sayce

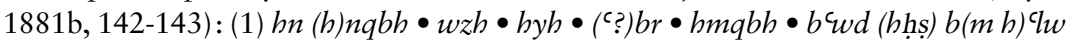

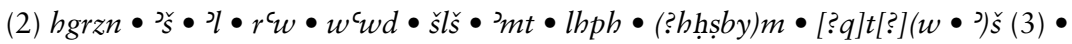
$\jmath \bullet r^{c} w \bullet m[d ?] h \bullet y\left[?^{c}\right] z d h \bullet b s q r \cdot m b \bullet m n \bullet q m(h \bullet w y) h k w \bullet b y r h \bullet b^{2}(4) n q b[h$ ? ]

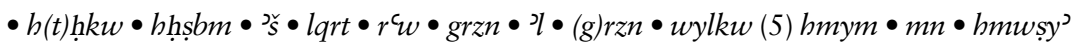

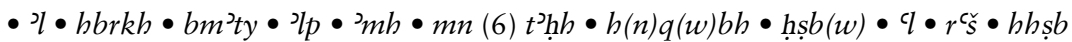
$z(b)$. "Behold the excavation! Now this is the further side (or the history) of the tunnel. While the excavators were lifting up the pick, each towards his neighbour, and while there were yet three cubits to the mouth (of the tunnel) the excavators were hewing. Each came to his neighbour at a measure's length (?) [...] in the rock on high; and they worked eagerly at (the) castle they had excavated (?); the excavators worked eagerly each to meet the other, pick to pick. And the waters flowed from their outlet at the Pool for a distance of a thousand cubits, from the lower part (?) of the tunnel (which) they excavated at the head of the excavation here» (p. 148-149).

16. (1) hnqbh $\bullet w z h \bullet h y h \bullet d b r \bullet h n q b h \bullet b^{c} w d(2) h g r z n \bullet s_{s} \bullet ग \bullet r^{c} w \bullet w b c w d \bullet \check{s} l s ̌ s$ $\bullet{ }^{\prime} m t \bullet l h n{ }^{c} \bullet q l \bullet \jmath_{s} \bullet q(3) r^{2}>\bullet r^{c} w \bullet k y \bullet h y t \bullet z d h \bullet b s q r \bullet m y m \bullet n r m w b y m \bullet$ h (4) nqbh $\bullet h k w \bullet h h s ̣ b m \bullet ~_{s} \bullet l q r t \bullet r^{c} w \bullet g r z n \bullet q \bullet g r z n \bullet w y l k y(5) ~ h m y m \bullet m n$ $\bullet b m w s^{2} \bullet ग \bullet b b r k b \bullet b m^{2} t y m \bullet w^{2} l p \bullet{ }^{2} m b \bullet w m\left[{ }^{\prime}\right](6) t \bullet{ }^{2} m b \bullet b y h \bullet g b h \bullet h s r \cdot q$ - $r \check{s} \bullet b h s \underline{b}[m]$ (Kautzsch 1881, 264).

17. «In his perhaps not unnatural annoyance at the appropriation by an Englishman of an important inscription which he had regarded as the special property of the German Association, he has forgotten the courtesy due to a sister Society which has been in the field for years before the German Palestine Association was founded, as well as the candour and the fairness we might expect from a scholar» (Sayce 1881c, 282). 
esquisses sur papier et pris une empreinte en gypse, à partir desquelles, en s'aidant de l'observation de la pierre, il avait dressé un facsimilé de meilleure qualité que les précédentes copies ${ }^{18}$. Parallèlement, Sayce fera une nouvelle tentative de lecture dans la livraison d'octobre du Palestine Exploration Fund Quarterly de 1881, en prenant en compte certaines des corrections de Kautzsch (à partir du facsimilé de J. Euting) et la copie que le lieutenant Claude R. Conder ${ }^{19}$ et son collègue, le lieutenant R. E. Mantell, avaient faite sur place, le 15 juillet, et publiée dans le même fascicule ${ }^{20}$. W. T. Pilter et H. Guthe, en ont fourni un autre facsimilé. Enfin, en 1883, A. H. Sayce publiera une nouvelle traduction de l'inscription:

(1) [Behold] the excavation! Now this had been the history of the excavation. While the workmen were lifting up (2) the axe, each towards his neighbour, and while three cubits still remained to [cut through], [each heard] the voice of the other who called (3) to his neighbour since there was an excess in the rock on the right hand and on [the left]. And on the day of the (4) excavation, the workmen struck, each to meet his neighbour, axe against axe, and there flowed (5) the waters from the spring to the pool for a thousand two hundred cubits; and [...] (6) of a cubit was the height of the rock over the heads of the workmen. (Sayce 1883, 210)

La nouvelle de la découverte de l'inscription parvint aussi en France, à l'Académie des Inscriptions et Belles-Lettres, qui s'en était fait l'écho par l'entremise de Jean Derenbourg' ${ }^{21}$. Celui-ci regrettait que la France fût

18. La photographie du moulage en gypse accompagne l'article de Guthe de 1882 (Guthe 1882, en fin de volume), mais une copie (bien imparfaite) réalisée par l'orientaliste suisse A. Socin d'après le moulage en gypse avait déjà été publiée par Kautzsch dans Zeitschrift der Deutschen Morgenländischen Gesellschaft 4/3-4, pl. 8.

19. Alors en charge de la Survey of the Eastern Palestine par la Palestine Exploration Fund.

20. Conder avait vu l'inscription grâce à Guthe et établi son dessin en s'aidant des notes et esquisses de ce dernier (Conder 1881b, 286-292; Zeitschrift der Deutschen Morgenländischen Gesellschaft 1880, 54; Palestine Exploration Fund Quarterly 1880, 238). Sayce: «(1) Behold the excavation! Now this is the history of the tunnel. While the excavators were lifting up (2) the pick, each towards the other, and while there were yet three cubits to be broken through [...] the voice of the one called (3) to his neighbour, for there was an excess (?) in the rock, on the right. They rose up... they struck on the west of the (4) excavation, the excavators struck, each to meet the other, pick to pick. And there flowed (5) the waters from their outlet to the Pool for a distance of a thousand cubits; and (three-fourths ?) (6) of a cubit was the height of the rock over the head of the excavation here» (Sayce 1881c, 282-285).

21. Lors de la séance du $1^{\text {er }}$ avril 1881 , puis le 23 et le 30 septembre de la même année (Comptes Rendus de l'Académie des Inscriptions et Belles-Lettres 25/2, 1881, 70). 
absente des débats qui opposaient alors les savants allemands et anglais, qui rivalisaient dans la quête de la moindre inscription relative à la Bible. En effet, le jeune orientaliste et diplomate Charles Clermont-Ganneau (18461923), nommé vice-consul de France à Jaffa ${ }^{22}$, fut empêché de se rendre à Jérusalem par le typhus qu'il avait contracté dès son arrivée à Jaffa, en janvier $1881^{23}$. S'il fit part au ministre de son intention d'entreprendre le voyage à Jérusalem pour étudier l'inscription ${ }^{24}$, il ne faisait pourtant pas la moindre allusion à l'inscription hébraïque du tunnel de Siloé dans sa lettre envoyée à l'Académie des Inscriptions et Belles-Lettres, datée du 16 juil$\operatorname{let}^{25}$.

Au mois d'août 1881, à Jérusalem, Clermont-Ganneau étudiait enfin sur place et prenait des estampages et des moulages de l'inscription. Le 12 septembre 1881, après un mois de campagne à Jérusalem, il écrivait, dans une lettre adressée à Ernest Renan (Clermont-Ganneau 1881), avoir «fait plusieurs longues séances dans l'aqueduc », et en avoir rapporté "des estampages et d'excellents moulages ${ }^{26}$. Il pensait «avoir réussi à lire

Derenbourg s'était contenté en un premier temps de citer l'étude de l'inscription faite par Sayce (Derenbourg 1881a), avant de proposer une transcription complétée par des restitutions de lettres, suivie d'un premier essai de traduction française (Derenbourg 1881b), le tout fondée sur une photographie de l'inscription qui n'a été présentée qu'au cours de la séance suivante, le 30 septembre 1881 (Comptes Rendus de l'Académie des Inscriptions et Belles-Lettres 25/3, 1881, 166).

22. Correspondant de l'Académie des Inscriptions et Belles-Lettres; des missions archéologiques lui seront confiées également par la Palestine Exploration Fund en 18731874 .

23. Voir son premier rapport sur la mission (Clermont-Ganneau 1882, 5-10).

24. Par lettre datée du 13 juin 1881.

25. Comptes Rendus de l'Académie des Inscriptions et Belles-Lettres 25/3, 1881, 186187.

26. La gravure héliographique de la planche VIII de Clermont-Ganneau (1882b) a été réalisée par M. Perrot d'après les moulages envoyés à Paris en 1882 pour le Corpus inscriptionum semiticarum et pour le musée du Louvre (le cliché héliographique pris du moulage est signé par l'atelier Dujardin). "J'en ai pris d'excellents moulages, que je vous ai envoyés, Monsieur le Ministre, à la date du 24 courant [septembre 1881], pour être transmis à la Commission chargée par l'Académie des inscriptions et belleslettres» (Clermont-Garneau 1882a, 46: Quatrième rapport.). Le Cinquième rapport fait état de « 1 copie, 2 estampages, 3 moulages en plâtre» (1882b, 203, nr. 20). La note précise que deux des trois moulages avaient été envoyés à Paris, au Ministère de l'instruction publique pour être transmis à l'Académie pour la Commission du Corpus inscriptionum semiticarum. Voir aussi Clermont-Ganneau $(1888,293)$. 
l'inscription en totalité, au moins pour les parties où elle n'est pas irrémédiablement détruite ${ }^{27}$. Clermont-Ganneau rendit publiques les résultats de ses investigations dans un mémoire lu devant l'Académie en 1882, à son retour de Palestine ${ }^{28}$.

Malmenée par la suite, victime de sa célébrité, alors que sa lecture se précisait, l'état de l'inscription se dégrada brutalement et de manière irréversible lors d'une excision, qui prit par surprise le monde scientifique, en 1890. La quête de l'objet soustrait à son emplacement d'origine retint l'attention des autorités et celle des savants, trop émus par sa disparition pour soupçonner un confrère d'être à l'origine de ce larcin épigraphique.

Déjà en 1881, dans une lettre adressée à Ernest Renan, Clermont-Ganneau affirmait que, ne pouvant pas y prendre de photographie au magnésium, faute de recul suffisant, il avait persuadé les Turcs à exciser l'original, sans mesurer les risques courus ni craindre pour l'intégrité de l'inscription lors d'une éventuelle extraction: "J'ai mis en tête aux Turcs de faire exciser l'original, voyant que je ne pourrais en obtenir l'autorisation pour moimême. Le pacha en a référé à Constantinople. Quand l'opération sera faite et le texte amené au jour, j'en prendrai une belle photographie à votre intention " (Clermont-Ganneau 1881, 251-252; je souligne). Dans sa lettre adressée le 13 juin 1881, Clermont-Ganneau avait fait part à son ministre de ce même projet:

Je tenterai même d'obtenir du pacha de Jérusalem l'autorisation de faire exciser l'inscription dans le roc vif, comme je l'ai fait autrefois pour les deux inscriptions de la vallée de Josaphat dont je parle plus haut et pour les inscriptions marquant le périmètre sacré de Gezer. Dans ce cas, je ferai tout mon possible pour que la possession de ce précieux monument nous soit accordée. (Clermont-Ganneau 1882a, 9$)^{29}$

27. Voir aussi Quatrième rapport (Clermont-Ganneau 1882a, 46). Il semble faire allusion aux polémiques qui se sont intensifiées lorsque, en 1881, l'inscription avait été nettoyée à l'aide d'acides censés éliminer des interstices les dépôts minéraux. Cela fut vivement critiqué par Pilter et Sayce qui prétendaient que l'acide avait endommagé l'inscription ainsi que les nettoyages réitérés par Guthe, ce que Conder semblait infirmer (Sayce 1881c, 282; Conder 1882, 123).

28. Il y déchiffra notamment la mention de la longueur du canal: mille deux cents coudées $\left(b m^{2} t y m w^{\top} l p\right)$. En 1888, il donna une série de conférences à l’École Pratique des Hautes Études, consacrée à l'étude de l'aqueduc et de l'inscription.

29. En effet, deux autres inscriptions hébraïques trouvées par lui en 1870, incisées dans le roc d'une tombe du village de Silwan, avaient également été excisées sous ses ordres et envoyées au British Museum. 
L'inscription fut donc excisée à la grande indignation de la communauté scientifique anglaise et allemande, qui ne désignera pas comme instigateur de ce détournement Clermont-Ganneau, acquis à la sympathie des orientalistes de tous bords après la découverte de la stèle de Mésha et la dénonciation des fraudes épigraphiques. Le monde scientifique anglosaxon n'avait pas prêté attention à ses aveux lointains et les chercheurs français qui se sont par la suite penchés sur l'inscription ont négligé ce qui ne paraissait qu'un simple incident de parcours dans l'histoire des découvertes épigraphiques ${ }^{30}$.

Pouvait-il, Clermont-Ganneau, espérer que l'opération d'excision de l'inscription de Siloé allait être plus réussie, vingt ans après la récupération des bris de la stèle de Mésha? Le Palestine Exploration Fund avait exprimé ses « regrets et l'indignation» et dénonçait à juste titre un acte de vandalisme, proposant de collaborer avec les autorités pour retrouver la trace de l'inscription ${ }^{31}$. L'enquête menée par les autorités ottomanes pour retrouver l'objet fit de son excision un acte passible de poursuites judiciaires. D'après les résultats des investigations, l'inscription ainsi extraite de son site avait d'abord été vendue par un Fellah (à l'identité tue) à un marchand grec ${ }^{32}$. Retrouvée, l'inscription a été transférée au musée de Constantinople où elle

30. La stèle moabite de Mésha, découverte à Dhibân en août 1868 par le missionnaire protestant F. A. Klein, subit un sort semblable. Les tractations engagées par ClermontGanneau par l'intermédiaire du cheïkh Id el-Faîz n'ont abouti qu'à déterminer les Beni-Hamîdé, propriétaires des lieux et intrigués par l'intérêt porté par les Occidentaux à ce bloc de pierre, de faire éclater la stèle en basalte noir par un procédé thermique, afin de vérifier si elle ne dissimulait pas quelque trésor. Ce fut grâce à ce document que Clermont-Ganneau, alors drogman chancelier ad interim à Jérusalem, avait percé les milieux scientifiques des orientalistes. Quant au reproche qui pouvait lui être fait, d'avoir été à l'origine de la destruction de la stèle, il peut être déduit des propos qui se voulaient rassurants de la part de ses protecteurs, Ernest Renan et Melchior de Vogüé: «Ce qui est publié suffit pour le moment, et votre gloire est assurée; les brochures pleuvent d'Allemagne, de Russie, etc., et il n'est question que de vous "; «Ne vous troublez pas des chicanes qu'on vous fera. Personne ne vous enlèvera la gloire d'avoir découvert un monument unique» (extraits de lettres de M. de Vogüé [18 avril 1870] et d'E. Renan [18 mars de la même année], rapportés dans DupontSommer 1974, 596).

31. Lettre du $1^{\text {er }}$ novembre 1890 et Fund's Office 195/1727, nr. 3 ; note dans le Palestine Exploration Fund Quarterly Statement de janvier 1891, p. 2; lettre du 18 février 1891, du proconsul britannique de Jérusalem, William H. Khayat à Sir William H. White, membre de la Royal Society. En même temps, le Comité dénonçait une fausse inscription présentée comme provenant du tunnel de Siloé (p. 3) et dont des copies semblent avoir circulé en Angleterre. Voir aussi Guthe (1890, 203-204).

32. Georgios Patrides (Palestine Exploration Fund Quarterly 1891, 2, infra). 
restera et la recherche des responsabilités fut abandonnée ${ }^{33}$. Comme l'attestent les premiers facsimilés, alors qu'elle était encore in situ, l'inscription était déjà traversée par une fracture à gauche, affectant les trois premières lignes. Lors de son extraction, la pierre s'était brisée en deux grands morceaux, en suivant l'ancienne fracture. Les premiers facsimilés et transcriptions devenaient dès lors précieux.

\section{Le tunnel de Siloé, œuvre du roi Ézékias ?}

L'on s'accordât, et ce dès les premières investigations et avant même la découverte de l'inscription en 1880, pour reconnaître dans le tunnel l'ouvrage d'art et les travaux hydrauliques à Jérusalem du roi Ézékias. Les sources bibliques et les écrits de Flavius Josèphe témoignent de l'existence d'installations hydrauliques à Jérusalem, dont l'origine était située à haute époque. "Quiconque veut frapper le Jébusite, doit atteindre le canal ( $k o l$ makkēh yəbusî woyigga caș̣sinnôr) », peut-on lire dans 2 Sam 5,834. Voulait-on suggérer par-là la possibilité d'une prise de la ville en en obstruant les conduits d'eau ou bien que l'on pouvait y pénétrer par les égouts ${ }^{35}$ ? Des travaux d'adduction d'eau attribués au roi de Juda, Ézékias, sont par ailleurs mentionnés allusivement dans 2 Rois 20,20: «[...] ce qu'il [Ézékias] a fait, le réservoir et le canal construits pour amener l'eau dans la ville ('et

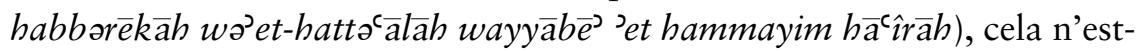

33. Une note de la livraison d'avril 1891 du Palestine Exploration Fund Quarterly (p. 89) rapporte une information donnée par Henry Gillman qui a vu les fragments de l'inscription chez le gouverneur de Jérusalem (Ibrahim Hakki Pacha, gouverneur de Jérusalem de novembre 1890 à novembre 1897), avant qu'ils soient acheminés vers Constantinople.

34. Le mot șinnôr, évoquant en premier lieu les bruits d'une chute d'eau ou d'un écoulement, est ici compris comme "canal ", sens déduit à l'aide du contexte de Ps 42,8 où ce terme apparaît également et où il est question du bruit des eaux. Si Jérôme traduit șinnôr par fistula, "conduit », la Septante l'avait compris tout autrement, le tradui-

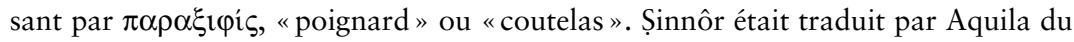

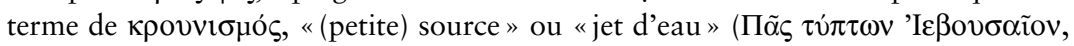

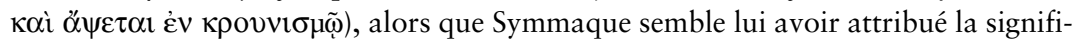

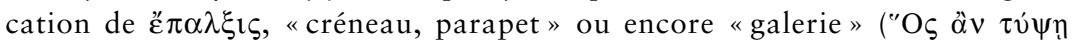

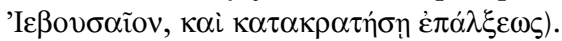

35. Vincent $(1912,148)$ cite, à titre d'exemple, le récit que donne Virgile du rapt du Palladium de Troie par Ulysse et Diomède (Æneida 2, 162-170), introduits dans la ville par les égouts, ainsi que le commentaire de Servius au vers 164: Tunc Diomedes et Ulixes, ut alii dicunt cuniculis, ut alii cloacis, ascenderunt arcem et occisis custodibus sustulere simulacrum (Maurus Servius Honoratus. In Vergilii carmina comentarii, recensuerunt, G. Thilo et H. Hagen, dir., Leipzig, Teubner, 1881). 
il pas écrit dans le livre des Annales des rois de Juda?». À plus de quatre siècles d'écart, le Siracide mentionnait les travaux d'adduction d'eau attribués à ce roi: «Ézékias fortifia sa ville, en y amenant l'eau à l'intérieur. Avec le bronze, il creusa le rocher et construisit des réservoirs pour les eaux. De son temps monta Sennachérib qui envoya Rab-Šāqēh [... ${ }^{36} »($ Si 48,17-18). Selon 2 Chr 32,3-5, Ézékias avait bloqué toutes les sources qui se trouvaient à l'extérieur de Jérusalem:

Il [Ézékias] se concerta avec ses dignitaires et ses officiers pour obturer l'accès à l'eau des sources situées en dehors de la ville (listôr 'et-mêmēy

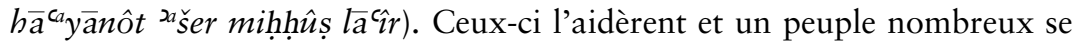
rassembla pour obturer toutes les sources et le ruisseau coulant à l'intérieur de la terre (wayistomû 'et-kol-hamma yānôt wo'et-hannahal haššôțēp botôk$h \vec{a} \bar{a} r e s$ ), en disant: "Pourquoi les rois d'Assyrie, à leur arrivée, trouveraient-ils de l'eau en abondance? " Ézékias se mit courageusement à reconstruire tout le rempart démoli, il construisit les tours et une autre à l'extérieur du mur (wayyiben 'et-kol-haḥhômāh happorûsāah wayya'al 'al hammigdālôt wolaḥhûșāh hạ̣hômāh 'aḥeret).

Le traducteur de la Septante comprend différemment ce passage, puisqu'il semble s'agir non plus d'une tour se trouvant à l'extérieur de la muraille, mais d'un deuxième rempart: "et il construisit tout le mur qui avait été démoli, les tours, ainsi qu'un autre [mur] en face, à l'extérieur »

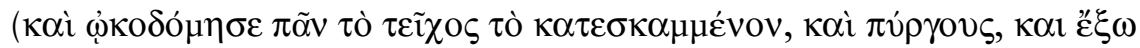
$\pi \rho \circ \tau \varepsilon \dot{\chi} \chi(\sigma \mu \alpha \ddot{\alpha} \lambda \lambda \mathrm{o})$. L'interprétation de la Septante resterait simplement anecdotique si elle ne concordait pas avec un passage de Is 22,11 , où il est question de «deux murailles»: "Vous avez aménagé un bassin entre les deux murailles pour les eaux de l'ancien réservoir ( $\hat{u} m i q w \bar{a} b^{{ }^{c}}{ }^{\prime} \hat{i} \hat{t} e m ~ b \bar{e} y n$ haḥhōmōtayim lomēy habborēkāh hayəšānāh)».

Selon Flavius Josèphe, le réservoir de Siloé (qu'il appelle «source», $\pi \eta \gamma \eta \dot{)}$ ) se serait trouvé à proximité de l'ancienne enceinte ${ }^{37}$, mais selon

36. D'après l'édition du texte hébreu établie par I. Lévi: yhaqqyhw haq 'yrw bhṭt ' mym wyḥ̣sb knḩšt șwrym wyḥswm hrym mqwh bymyw lh snhryb wyšlh 't rb šqh...

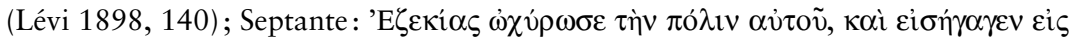

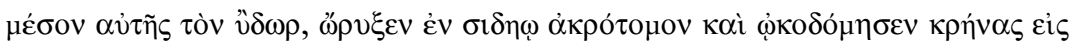

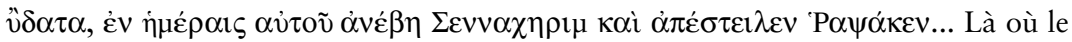
texte hébraïque mentionne le «bronze », le traducteur de la Septante opte pour le «fer».

37. Bell. V, 140 (Flavius Josèphe 1982): «quant au ravin dit "des fromagers", dont nous avons vu qu'il séparait la colline de la ville haute de la colline inférieure, il descend jusqu'à Siloé; car c'est ainsi que nous appelons cette abondante source d'eau douce »; 
Birch, il ne faisait pas de doute que la piscine de Siloé se trouvait entre les deux murailles évoquées en Is 22,11, et en deçà du «mur extérieur », mentionné dans 2 Par 32,5 (Septante). S'agit-il du «réservoir inférieur» de Is 22,9 (habborēkāh hattạ̣tônāh) aménagé sous Akhaz, vers 735, (Birkat al-Hamra)? De l'avis de Birch, les deux murailles formaient le mur d'enceinte de la ville et le mur extérieur (2 Par 32,5), en plaçant ainsi la piscine de Siloé à l'intérieur de ces remparts (Birch 1880, 200), mais les discussions sur le tracé des murailles antiques à Jérusalem a fait l'objet d'interminables débats.

La paléographie de l'inscription hébraïque constitue un autre argument en faveur d'une datation dans la seconde moitié du viII ${ }^{\mathrm{e}}$ siècle qui vient compléter les informations des données bibliques et assyriennes sur le règne d'Ézékias. On a ainsi associé l'ouvrage d'art à la campagne de Sennachérib (704-681), qui avait mis le siège à Jérusalem en 701, date établie d'après les Annales royales assyriennes ${ }^{38}$.

La laborieuse percée du tunnel d'Ézékias exclut toutefois une entreprise hâtive. Si elle a bien eu lieu avant la campagne de Sennachérib, elle devait avoir débuté suffisamment à l'avance pour permettre l'achèvement des travaux, donc avant l'an 702. Or, la mort de Sargon II, en 705, avait jeté l'Assyrie dans une période de confusion, en même temps qu'avaient lieu des troubles en Babylonie, offrant par là une occasion propice à une éventuelle révolte d'Ézékias. De l'avis de W. Mayer, Ézékias pouvait alors entreprendre des travaux d'adduction d'eau dans la cité à l'occasion de ce changement de règne et en ne déclarant son insoumission, déclencheur de la campagne punitive de Sennachérib, qu'une fois les travaux terminés (Mayer 2003, 172-173).

La position de Dalley, consistant non seulement à minimiser l'agressivité assyrienne face à Juda, mais à voir dans la personne d'Ézékias non pas un révolté mais un allié, reste isolée ${ }^{39}$. Selon Dalley, Ézékias aurait même été une sorte d' «agent secret» de Sennachérib auprès des Philistins, et le

145: «[...] de là, au Sud, elle [l'ancienne enceinte] s'infléchissait jusqu'au-delà de la

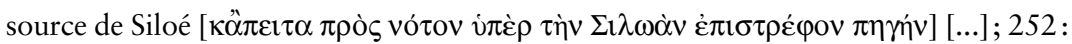
«[...] toute la portion de l'ancien rempart qui, de la piscine de Siloé, s'infléchit à l'Est et descend jusqu'au palais de Monobaze [...]»; 410: «[...] la source de Siloé avait tari ainsi que toutes celles qui sont hors la ville $[\ldots] »$.

38. Essentiellement les prismes Taylor et celle de Chicago, rédigées peu de temps après la campagne, en 691 et 689 respectivement (Mayer 2003).

39. D’après Dalley, Sennachérib aurait cherché à intimider Ézékias, sans intention de le tuer ni même de le capturer (Dalley 2004, 392). 
prince d'Ekron, Padi, enfermé à Jérusalem, aurait été non pas le captif d'Ézékias, mais son protégé. Sans pouvoir éluder les sources qui semblent infirmer une telle interprétation et l'exclure tout à fait ${ }^{40}$, Dalley voit dans la campagne de Sennachérib en Juda un siège passif plutôt qu'une vraie offensive assyrienne. Par conséquent, la construction du tunnel, que Dalley attribue au règne d'Ézékias, n'aurait pas eu pour but celui de prévenir un siège que le monarque pro-assyrien, selon cette interprétation, n'aurait pas eu à craindre ${ }^{41}$.

L'attribution de l'entreprise de la percée du tunnel de Siloé au règne d'Ézékias a été plus récemment contestée par Reich et Shukron, qui y voient plutôt un ouvrage d'art datant de la fin du Ix ${ }^{\mathrm{e}}$ siècle ou du début du $\mathrm{VIIII}^{\mathrm{e}}{ }^{42}$. Pour soutenir cette datation haute, les deux archéologues se fondent sur les fouilles récentes de la Cité de David, des parties nord du tunnel et sur la complexe configuration des canaux creusés près de la source du Ghihon (canaux I et II; tunnels III, IV [et la «Chambre ronde» du réservoir creusé dans le roc, découverte par la mission Parker-Vincent, en 1909] et VI) (Reich et Shukron 2011, 148-150). À cette théorie, Finkelstein oppose plusieurs arguments dont celui de l'extension de la ville à la colline sud-ouest, notamment sur son versant sud-est, dans la seconde moitié du viII ${ }^{\mathrm{e}}$ siècle, et plutôt dans les années 730 ou 720 qu'à la toute fin du VIII ${ }^{\mathrm{e}}$ siècle, datation qui serait confortée par les analyses de radiocarbone et de la poterie (Finkelstein 2013). Par conséquent, le tunnel n'aurait pas été taillé en prévision du siège de la ville par Sennachérib, mais quelques décennies plus tôt, pendant les règnes des souverains assyriens Tiglath-Pileser III (745727), Salmanasar V (727-722) ou Sargon II (721-705) et des rois judéens Akhaz ou Ézékias.

Concernant les dates du règne d'Ézékias, deux chronologies possibles sont avancées par les historiens. Si son règne avait débuté entre 729 et 727 et duré vingt-neuf ans (selon 2 R 18,2), il se serait achevé en 700/698, ce qui

40. Les prismes de Chicago, col. III, 1. 18-40; Taylor, col. III, 1. 11-33; Thompson, 1. 13-15; les bulles nr. 2, 3 et 4 (Mayer 2003, 186-200).

41. Il s'agirait, selon l'auteur, d'imiter l'œuvre de Sennachérib, et, par conséquent, d'une forme subtile de flatterie à l'égard du souverain assyrien (Dalley 2004, 398).

42. "The inevitable conclusion of this situation is that Tunnel IV, or the entrance to the northern part of the Siloam Tunnel, was dug at the very latest at the end of the 9th century or in the early part of the 8th century BCE. An unavoidable historical conclusion of this study is that the hewing of the Siloam Tunnel cannot be attributed to Hezekiah» (Reich et Shukron 2011, 154). 
ne serait pas sans poser des problèmes d'harmonisation avec $2 \mathrm{R}$ 18,1.910. Selon $2 \mathrm{R} 18,13$, où il est précisé que l'expédition de Sennachérib eut lieu la quatorzième année du règne d'Ézékias, le début du règne d'Ézékias serait alors à placer entre $716 / 714$ et sa fin entre $688 / 686$, sans tenir compte des synchronismes de 2 R 18,1.9-10, à moins de supposer qu’Ézékias a été associé au trône dès 728 (Gonçalves 1986, 51-60) ) $^{43}$. Cette possible association au trône avant le règne proprement dit pourrait alors concilier certaines données archéologiques, telles que Finkelstein les interprète, et les indications bibliques attribuant à Ézékias les installations hydrauliques, notamment le creusement dans le roc (Si 48,17-18, supra). Néanmoins, et ce sera la conclusion de cet article, la tenace tradition biblique qui les associe aux préliminaires de la campagne assyrienne de Sennachérib semble étayer une date plus tardive, aux débuts du règne de Sennachérib.

\section{Références}

Albright, W. F. (1955), «The Siloam Inscription», dans J. B. Pritchard, dir., Ancient Near Eastern Texts Relating to the Old Testament, Princeton, Princeton University Press, p. 321.

Amiran, R. (1975), "The water Supply of Israelite Jerusalem », Jerusalem Revealed. Archaeology in the Holy City 1968-1974, Jérusalem, The Israel Exploration Society, p. 75-84.

BeCKerath, J. von (1992), «Ägypten und der Feldzug Sanherib's im Jahre 701 v.Chr. ", Ugarit Forschungen, 24, p. 3-8.

Birch, W. F. (1880), "Gihon », Palestine Exploration Fund Quarterly Statement, p. 199-200.

(1884), «Notes on Præ-Exilic Jerusalem », Palestine Exploration Fund Quarterly Statement, p. 70-75.

Bliss, F. J. et Dickie, A. C. (1898), Excavation at Jerusalem, 1894-1897, Londres.

Clermont-Ganneau, C. (1881), «Nouvelles archéologiques et correspondances " [Lettre à E. Renan, datée du 12 septembre 1881], Revue archéologique, 42, octobre, p. 251-253.

43. Les remaniements du texte à l'époque perse pourraient expliquer certains anachronismes, comme par exemple la mention du pharaon nubien Tirhaqa/Taharqo (XXV dynastie) en 2 R 19,9, dont le règne débute en 690/689 (Beckerath 1992, 3-4). 
(1882a), Premiers rapports sur une mission en Palestine et en Phénicie entreprise en 1881 (Archives des missions scientifiques et littéraires série 3, t. 9), Paris, Imprimerie nationale.

(1882b), Rapports sur une mission en Palestine et en Phénicie entreprise en 1881 (Archives des missions scientifiques et littéraires série 3, t. 9), Paris, Imprimerie nationale.

(1888), «L'inscription hébraïque de l'aqueduc de Siloé », Recueil d'archéologie orientale, 1, E. Leroux, Paris, p. 293-299; pl. XVI.

(1897), «Les tombeaux de David et des rois de Juda et le tunnelaqueduc de Siloé", Comptes Rendus de l'Académie des Inscriptions et Belles-Lettres, 41/4, séance du 13 août, p. 383-427.

Conder, C. R. (1881a), «Lieutnant Conder's Reports: Siloam», Palestine Exploration Fund Quarterly Statement, juillet, p. 197-199.

(1881b), "The Siloam Inscription ", Palestine Exploration Fund Quarterly Statement, octobre, p. 288-292.

(1882), «The Siloam Tunnel », Palestine Exploration Fund Quarterly Statement, avril, p. 122-131.

Dalley, S. (2004), «Recent Evidence from Assyrian Sources for Judaean History from Uzziah to Manasseh ", Journal for the Study of the Old Testament, 28/4, p. 387-401.

Derenbourg, J. (1881a), "L'inscription du tunnel près de la fontaine de Siloé, à Jérusalem ", Comptes Rendus de l'Académie des Inscriptions et Belles-Lettres, 25/2, p. 97-100.

(1881b), «Inscription hébraïque du tunnel près de la fontaine de Siloé, à Jérusalem ", Comptes Rendus de l'Académie des Inscriptions et Belles-Lettres, 25/3, p. 199-205.

Diringer, D. (1934), Le iscrizione antico-ebraiche palestinesi, Florence, Felice Le Monnier.

Dupont-Sommer, A. (1974), "Un dépisteur de fraudes archéologiques: Charles Clérmont-Ganneau (1846-1923), membre de l'Académie des Inscriptions et Belles-Lettres ", Comptes rendus de l'Académie des Inscriptions et Belles-Lettres, 118/4, p. 591-609.

FABricius, E. (1884), "Alterthümer auf der Insel Samos », Mittheilungen des deutschen archäologischen Institutes in Athen, 9, p. 163-197.

Feyel, M. (2000), "Comment restituer le dioptre d'Héron d'Alexandrie? », Autour du dioptre de Héron d'Alexandrie (Mémoires 21, Centre Jean Palerne), Saint-Étienne, Publications de l'Université de Saint-Étienne, p. 191-226. 
Finkelstein, I. (2013), "The Finds from the Rock-Cut Pool in Jerusalem and the Date of the Siloam Tunnel. An Alternative Interpretation", Semitica et Classica, 6, p. 279-284.

Fischer, A. (1902), "Zur Siloahinschrift », Zeitschrift der Deutschen Morgenländischen Gesellschaft, 56, p. 800-809.

Flavius Josèphe (1982), Guerre des Juifs, t. 3 / trad. par A. Pelletier, Paris, Les Belles Lettres.

Frumkin, A. et Shimron, A. (2006) «Tunnel engineering in the Iron Age: geoarchaeology of the Siloam tunnel, Jerusalem ", Journal of Archaeological Science, 32/2, p. 227-237.

Gonçalves, F. J. (1986), L'expédition de Sennachérib en Palestine dans la littérature hébraïque ancienne, Paris, Gabalda.

Guthe, H. (1881), "Über die Siloahinschrift ", Zeitschrift des Deutschen Palästina-Vereins, 4, p. 250-259.

(1882), «Die Siloahinschriften », Zeitschrift der Deutschen Morgenländischen Gesellschaft, 36, p. 725-750.

(1890), «Die echte und die gefälschte Siloah-Inschrift », Zeitschrift des deutschen Palästina-Vereins, 13, p. 203-204; "Das Schicksal der Siloah-Inschrift », p. 286-288.

Kautzsch, E. (1881), "Die Siloahinschrift», Zeitschrift des Deutschen Palästina-Vereins, 4, p. 102-114; 260-272.

LÉvi, I. (1898), dir. et trad., L'Ecclesiastique ou la Sagesse de Jésus, fils de Sira, Paris, Ernest Leroux.

Mayer, W. (2003), "Sennacherib's Compaign of 701 вCE: The Assyrian View », dans L. L. GrabBe, dir., 'Like a Bird in a Cage'. The Invasion of Sennacherib in 701 всE, Londres/New York, Sheffield Academic Press (JSOT Supplement Series 363).

ReICH, R. (2011), Excavating the City of David. Where Jerusalem History's began, Jérusalem, Israel Exploration Society.

Reich, R. et Shukron, E. (2002), «Reconsidering the Karstic Theory as an Explanation to the Cutting of Hezekiah's Tunnel in Jerusalem », Bulletin of the American Schools of Oriental Research, 325, p. 75-80.

(2011), "The Date of the Siloam Tunnel Reconsidered ", Tel Aviv, 38 , p. 147-157.

Robinson, E. et Smith, E. (1841), Biblical Researches in Palestine, Mount Sinai and Arabia Petraea: A Journal of Travels in the Year 1838, 3 vol., Boston. 
Sayce, A. H. (1881a), "The Inscription at the Pool of Siloam», Palestine Exploration Fund Quarterly Statement, avril, p. 69-73.

(1881b), «The Ancient Hebrew Inscription Discovered at the Pool of Siloam in Jerusalem ", Palestine Exploration Fund Quarterly Statement, juillet, p. 141-154.

(1881c), "The Ancient Hebrew Inscription in the Pool of Siloam», Palestine Exploration Fund Quarterly Statement, octobre, p. 282-285.

(1883), «The Siloam Inscription", Palestine Exploration Fund Quarterly Statement, juillet, p. 210-215.

Schick, C. (1878), «Die Wasserversorgung der Stadt Jerusalem », Zeitschrift des Deutschen Palästina-Vereins, p. 137-176.

(1880), «Phœnician Inscription in the Pool of Siloam», Palestine Exploration Fund Quarterly Statement, octobre, p. 238-239.

Socin, A. (1899), Die Siloahinschrift zum Gebrauch bei akademischen Vorlesungen, Mohr, Freiburg.

Spon, J. (1679), Miscellanea eruditae antiquitatis sive supplementi gruteriani. Liber primus, Francfort/Venise, J. H. Viderholdt/F. Rota.

Tobler, T. (1853), Denkblätter aus Jerusalem, Sankt Gallen und Konstanz. (1858), Planography of Jerusalem. Memoir to Accompany the New Ground-Plan of the City of Jerusalem and the Environs, constructed anew by C. W. M. Van de Velde after the plans of the Engineers Aldrich and Symonds, and of Dr. Tobler, Gotha/Londres, Justus Perthes/ Williams \& Norgate.

V[InCENT], H. (1911), Underground Jerusalem: Discoveries on the Hill of Ophel (1909-1911), Londres.

VinCENT, H. (1912), Jérusalem. Recherches de topographie, d'archéologie et d'histoire. I. Jérusalem antique, Paris, Gabalda.

Vincent, L.-H. et Steve, A.-M. (1954), Jérusalem de l'Ancien Testament. Recherches d'archéologie et d'histoire, $1^{\text {re }}$ partie et vol. de planches, Paris, Gabalda.

Warren, C. (1876), Underground Jerusalem. An Account of some of the Principle Difficulties Encountered in its Exploration and the Results Obtained, with a Narrative of an Expedition through the Jordan Valley and a Visit to the Samaritans, Londres, Bentley.

Wilson, C. W. (1865), Ordnance Survey of Jerusalem, Londres [H. M. Stationery Office, G. E. Eyre, W. Sopttiswoode]. 
Wilson, C. et Warren, C. (1871), The Recovery of Jerusalem. A Narrative of Exploration and Discovery of the City and the Holy Land, Londres, Bentley.

WeIL, R. (1947), La cité de David II. Campagne de 1923-1924, Paris.

\section{Résumé}

L'histoire de la découverte à Jérusalem d'un système hydraulique complexe sous la colline méridionale où fut creusé le tunnel de Siloé et de l'une des inscriptions hébraïques de la plus haute importance pour cette région avare en documents de nature épigraphique illustre de quelle manière une série de faits, dont certains fortuits, s'est posée à l'origine d'une reconstitution qui a mobilisé et continue de le faire des spécialistes du terrain et ceux du texte biblique, en brisant les barrières disciplinaires.

\section{Abstract}

This paper deals with the excavations' history of the Siloam tunnel on the Southern hill of Jerusalem, and the record of one of the most important Hebrew inscriptions. This major epigraphical discovery as well as the tunnel survey illustrate in which manner a series of material facts, some fortuitous, was behind the reconstruction of the past, which mobilized in a concerted way archaeologists and specialists of the biblical text until today, thus breaking the disciplinary barriers. 